\title{
On tonal pattern perception in monkeys (Cebus apella)
}

\author{
M. R. D'AMATO and MICHAEL COLOMBO \\ Rutgers-The State University, New Brunswick, New Jersey
}

\begin{abstract}
The monkey's capacity to extract tonal pattern from a sequence of tones was assessed in four subjects that had the benefit of substantial past experience in discriminating, matching, and remembering acoustic stimuli. In Experiment 1, the monkeys failed to transfer their wellestablished matching behavior to the matching of two structured sequences of tones that differed primarily in tonal pattern, indicating that for them tonal pattern was not a salient feature of the acoustic stimuli. Experiment 2 was an attempt to encourage tonal pattern perception by employing, within a discrimination paradigm, very simple tonal patterns and multiple exemplars of the positive and negative patterns; the transfer design, borrowed from Hulse and Cynx (1985), was a powerful one for revealing tonal pattern perception. Verifying earlier results from our laboratory, there was little in the monkeys' transfer performance to indicate that they had extracted tonal pattern from the acoustic stimuli. Major discriminative control seemed to be vested in the first tone of each exemplar. This apparent cognitive limitation may be rather general among animals, perhaps reflecting an intimate connection between the capacity for tonal pattern perception and that for acoustically based language.
\end{abstract}

Although there are notable exceptions (e.g., Cheney, Seyfarth, \& Smuts, 1986; Seyfarth, Cheney, \& Marler, 1980), most of our knowledge about the cognitive capacities of monkeys is based on the visual modality. In large measure this dependence on vision is due to the fact that monkeys ordinarily learn simple and complex relations based on non-species-specific stimuli far faster with visual discriminative cues than with acoustic cues (see D'Amato \& Salmon, 1982). Exploring the possibility that this asymmetry might be due in part to the custom of using relatively simple acoustic stimuli, D'Amato and Salmon $(1982,1984)$ assessed the monkey's ability to process complex acoustic stimuli, structured sequences of tones referred to as "tunes." The issue at stake in these studies was whether the monkey was capable of detecting the tonal pattern of such a sequence of tones-something that humans, even young children, accomplish with ease. By tonal pattern we mean the pattern of frequency (or pitch) increases and decreases described by a tune's sequence of tones-its frequency (or pitch) contour.

Although the initial results were promising (D'Amato \& Salmon, 1982), a detailed follow-up series of experiments was unambiguously negative (D'Amato \& Salmon, 1984). In brief, monkeys were trained with two 7-tone sequences that were very similar except for their tonal patterns. Although the monkeys learned with extended training to discriminate between the tunes, they failed to maintain the discrimination when both tunes were trans-

The present research was supported by National Science Foundation Grant BNS-8417383. The major findings of Experiments 1 and 2 were briefly described in D'Amato (1988). Address correspondence to $M$. R. D'Amato, Psychology Department, Rutgers-The State University, New Brunswick, NJ 08903. posed up or down by one octave, suggesting that they might have based their discrimination on acoustic features other than frequency contour. A long series of tests involving a variety of transformations of the tunes confirmed this suspicion. It turned out that the main discriminative burden was carried by the last note of the tunes, which alone could completely sustain the discrimination.

The failure of the monkeys to extract tonal pattern from the two training tunes despite extensive exposure to them might have been due to the fact that the monkeys had no previous experimental experience discriminating nonspecies-specific acoustic stimuli. Because of our concern that previous training with visual stimuli might inhibit monkeys from attending to auditory cues, we went to some length to obtain experimentally naive subjects. Although the absence of prior training with visual stimuli probably facilitated the monkeys' attending to relatively simple properties of the tunes, such as the frequency (or pitch) of individual notes, it seems possible that attending to more complex features of these auditory stimuli, in particular to the tunes' tonal patterns, might have been impeded by the monkeys' lack of previous discriminative experience with non-species-specific acoustic stimuli.

\section{EXPERIMENT 1}

Our first experiment addressed this issue. Four monkeys were available that had been successfully trained to match non-species-specific acoustic stimuli in an identity matching paradigm. Moreover, they were able to match novel acoustic stimuli at performance levels that indicated representation of the matching concept in the auditory modality (D'Amato \& Colombo, 1985). We reasoned that 
if these monkeys could detect the tonal patterns of the two tunes used by D'Amato and Salmon (1984), they should display substantial transfer when required to match the two 7-tone tunes.

Of course the latter result would also be obtained if the monkeys could easily differentiate the tunes by means of cues other than tonal pattern. However, the tunes had been constructed in such a way as to diminish this possibility: their average frequency was very similar, four of the seven notes were represented in both tunes, and the first note of the two tunes was identical. Successful matching of the two tunes would therefore strongly imply that the differentiating acoustic characteristic was frequency contour. On the other hand, failure to transfer would constitute evidence that the tonal pattern of the tunes was not a salient cue for the monkeys.

\section{Method}

\section{Subjects}

Four laboratory-born Cebus apella monkeys, three males (Boris, Tiny, and Tom) and one female (Moe), 52 to 63 months of age at the start of the study, served as subjects. ${ }^{1}$ They all had a variety of previous training with visual and auditory stimuli, the most recent being that reported in D'Amato and Colombo (1985). Having served in Experiments 2 and 3 of the D'Amato and Salmon (1984) study, Tiny had a good deal more auditory discriminative experience than the other subjects, who had participated in the much shorter and less demanding discriminative task of Experiment 1. Between 3 and 13 weeks elapsed between the completion of the D'Amato and Colombo (1985) study and the beginning of the present one.

Throughout this and the following experiment the animals were housed individually and provided with a daily ration of Purina Monkey Chow \#5045 (usually 1 to $3 \mathrm{~h}$ following an experimental session), adjusted to the maximum amount that supported reliable performance in each monkey. The daily rations were sufficient to maintain the animals at $82-95 \%$ of their free-feeding body weights.

\section{Apparatus}

The experimental apparatus was the same as in D'Amato and Colombo (1985). The front wall of the experimental chamber accommodated two response levers which were separated by $43 \mathrm{~cm}$, each $22 \mathrm{~cm}$ above the chamber floor. A speaker $(10-\mathrm{W}, 10-\mathrm{cm}$, 8-ohm Realistic Model FE-103) was located directly below each lever. The right speaker presented the sample tune, whereas the comparison tune was presented by the left speaker. Illumination was provided by an overhead houselight, which could be turned on, off, or dimmed. Noyes banana-flavored pellets $(190 \mathrm{mg})$, delivered to a food cup located on the right wall of the chamber, served as reinforcers.

The acoustic stimuli employed in this and the following experiment were generated by a Commodore PET microcomputer, its signals amplified by the amplifier portion of an MTU D/A converter (Model K-1002); the output from the sound generator contained numerous harmonics of the fundamental frequencies. The two $7-$ note tunes were the same as those employed in D'Amato and Salmon (1984, Experiments 2 and 3). The notes of tune 1 formed a pattern of alternating increases and decreases in frequency: 1397, 1572, $1397,1572,1984,2083$, and $1046 \mathrm{~Hz}$. The pattern of tune 2 was distinctly different, beginning with a decrease in frequency, followed by a monotonic increase over the last five notes: 1397, 1173, $1316,1397,1572,1761$, and $1984 \mathrm{~Hz}$. All notes were of equal duration, one playing of a tune requiring about $1.3 \mathrm{sec}$. The intensity of the tunes was as reported in D'Amato and Salmon (1984,
Experiment 1). Presentation of trials events and data recording were accomplished by a PDP 8/e minicomputer and a Commodore microcomputer and disk units.

\section{Procedure}

The go/no-go matching procedure was as described in D'Amato and Colombo (1985). In brief, a trial began with illumination of the houselight, followed $2 \mathrm{sec}$ later by presentation of the sample tune via the speaker under the right lever. The first response to the right lever that occurred after the sample had played for 2 sec terminated the sample tune, extinguished the houselight, and initiated a 0.5 -sec delay interval. At the end of the delay, the houselight was illuminated and simultaneously the comparison tune was presented by the speaker under the left lever. Responses on the left lever were not effective for the first $0.8 \mathrm{sec}$ after the onset of the comparison tune, which continued playing until the left lever was pressed or an additional $3 \mathrm{sec}$ (the response "window") had elapsed.

Correct responses were defined as pressing the left lever during the 3-sec response window on matching trials and refraining from pressing on nonmatching trials. Correct responses on matching trials resulted in termination of the comparison sample, delivery of a reward pellet, and entry into the 20 -sec intertrial interval (ITI). The sequence was the same on correct nonmatching trials, except that the reward pellet was omitted.

Incorrect responses (failing to press the left lever during the 3sec response window on matching trials, or pressing on nonmatching trials), resulted in a 1-min timeout, signaled by dimming of the houselight, followed by entry into the ITI. Response latencies on the left lever were measured in units of $20 \mathrm{msec}$; a latency of $3 \mathrm{sec}$ was assigned on trials in which no response occurred during the 3-sec response window.

Since the monkeys had been away from the matching task for a number of weeks, prior to testing they were given a single refresher 48-trial session, using the familiar low and high tones of the D'Amato and Colombo (1985) study. The four subjects averaged 96.4\% correct responses on this retraining session. Four 48-trial transfer test sessions with the two tunes followed, one session each day.

\section{Results and Discussion}

The percentage of correct responses on the four transfer sessions is presented in Figure 1 for each subject. It is clear from the figure that the monkeys were unable to match the tunes to any substantial degree, and that they showed little, if any, improvement over the four test sessions. A t-test based on each subject's percentage of correct responses averaged over the four sessions indicated that the group's overall mean (53.1\%) did not differ significantly from chance expectation $[t(3)=1.98$, $p>.10]$. Nor were any of the individual-subject t-tests, based on the percentage of correct responses from each of the four test sessions, significant. The only statistically significant deviation from chance was produced by the data of the first session $[t(3)=2.65, p<.05]$. However, the highest score of this session was gotten by Tiny, who, in the D'Amato and Salmon (1984) study, had extensive experience in discriminating between the test stimuli of the present experiment, albeit in a condition where the notes of each tune were randomly permuted from one trial to the next. That Tiny's performance in the matching task was so poor (compared to his earlier performance) indicates that in the 18 months which intervened between the 


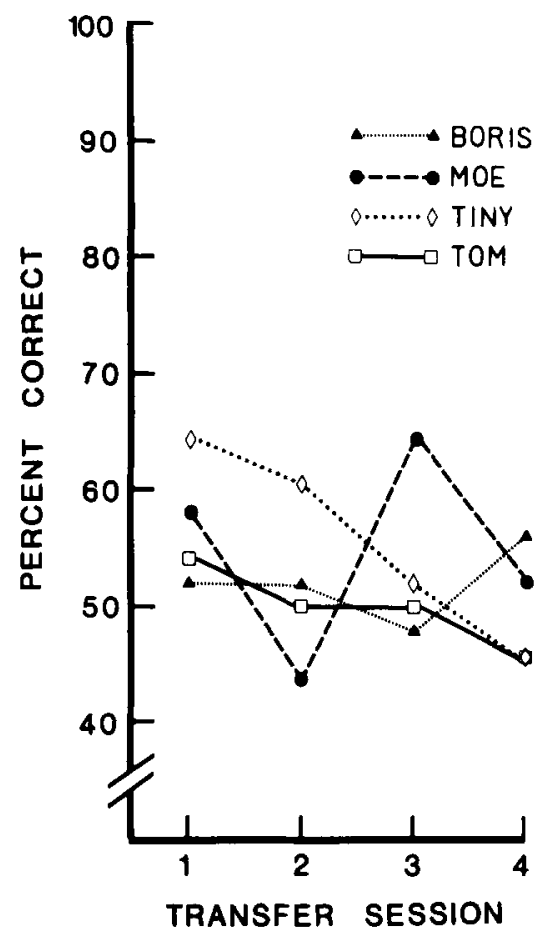

Figure 1. Percentage correct responses for each monkey during the four 48-trial matching sessions of Experiment 1. The two tunes of the D'Amato and Salmon (1984) study served as the sample set.

previous study and the present experiment the very difficult discrimination posed by the "random-notes" condition was largely lost.

To illustrate the degree of transfer achievable by the present subjects with new test stimuli that are discriminable on the basis of acoustic properties other than tonal pattern, over Tests 3-5 of the D'Amato and Colombo (1985) study, the four monkeys averaged $91.5 \%$ correct responses on the first transfer session and $95.3 \%$ correct on the second. The mean performance level of $57.3 \%$ correct attained in the first session of the present experiment, the highest reached in the four test sessions, pales by comparison.

In our go/no-go matching procedure, response latency is sometimes a more sensitive dependent variable than the percentage of correct responses (D'Amato \& Colombo, 1985). However, this did not apply to the present case. Group response latencies on matching and on nonmatching trials were not significantly different when the four test sessions were analyzed together in a randomizedblocks analysis of variance (ANOVA) $[F(1,21)<1]$. Nor were any of the four paired t-tests that evaluated response latency differences for each test session separately significant.

There is little indication from the results of Experiment 1 that extensive practice in discriminating among and matching a variety of acoustic stimuli improves the monkey's capacity to extract tonal pattern from nonspecies-specific acoustic signals. Given the present subjects' previous transfer accomplishments, the lack of im- provement in their matching performance over the four test sessions is especially discouraging. Still, the tonal patterns of the tunes, although easily differentiated by human adults, were rather complex. It is conceivable that simpler tonal patterns might fall within the monkeys' perceptual grasp. In Experiment 2, we explored this possibility.

\section{EXPERIMENT 2}

In addition to employing very simple tonal patterns, in Experiment 2 we utilized multiple exemplars of the positive and negative patterns. A plausible criticism of the D'Amato and Salmon (1984) study and of Experiment 1 is that a single exemplar of a tonal pattern might tend to focus the animal's attention on local acoustic cues, such as the absolute frequency of one or more of the individual notes. With multiple exemplars, frequency contour can be held constant while local cues are made to vary from one exemplar to another, which should set a premium on attending to tonal pattern.

The experimental design, devised by Hulse and Cynx (1985), was a powerful one for detecting tonal pattern perception in animals. The monkeys were first trained to discriminate among three exemplars of the positive tonal pattern and three exemplars of the negative pattern. The stimuli of this phase were contained within a "highoctave" frequency range. Next, the monkeys were trained with the same tonal patterns, but transposed two octaves down in frequency (the "low- $\propto$ ctave" frequency range). Inasmuch as our previous work showed little or no recognition of tonal patterns that were transposed only one $\alpha c-$ tave, we did not expect significant transfer to the low octave range. After being shifted between the high and low octaves, the monkeys received a series of critical test sessions in which the same tonal patterns were now located in the intermediate "middle" octave. If the monkeys had detected and employed the positive and negative tonal patterns in the high and low octave ranges, we thought they surely would show substantial transfer to these same patterns in the middle-octave frequency range.

\section{Method}

\section{Subjects and Apparatus}

The same four subjects as in Experiment 1 served in this study. During the approximately 18 months that separated the two experiments, the monkeys participated in an extensive study that assessed their short-term memory capacity for visual and acoustic stimuli (Colombo \& D'Amato, 1986). It is worth noting that their auditory short-term memory proved substantial, extending to retention intervals as long as $32 \mathrm{sec}$. Feeding and other maintenance conditions were the same as in Experiment 1, as was the apparatus.

There were six tones in the high octave range, approximately equally spaced on a logarithmic scale: $2688,3012,3378,3788$, 4237 , and $4808 \mathrm{~Hz}$. The three exemplars of the negative tonal pattern were 4-tone series of monotonically ascending frequencies, which began with the first, second, and third tones. They will be referred to as $A 1(2688,3012,3378$, and $3788 \mathrm{~Hz}), A 2(3012,3378$, 3788 , and $4237 \mathrm{~Hz}$ ), and $A 3(3378,3788,4237$, and $4808 \mathrm{~Hz})$. The three exemplars of the positive tonal pattern, D1, D2, and D3, 
consisted of the same three 4-tone series but played in the direction of descending frequencies. Thus, D1 was the sequence 3788 , 3378,3012 , and $2688 \mathrm{~Hz}$. Transposition to the low-octave frequency range was accomplished simply by dividing the frequency of each tone of a high octave exemplar by 4 ; in transposing to the middle octave range, the divisor was 2 . Each tone of an exemplar had an on-time of $140 \mathrm{msec}$ and an off-time of $80 \mathrm{msec}$, so that a single playing of an exemplar lasted $800 \mathrm{msec}$. Consecutive playings of an exemplar were separated by an 800 -msec off-period.

The monkeys' task, then, was to extract from the various exemplars whether frequency (or pitch) increased from one tone to the next or decreased-perhaps the simplest of frequency contour determinations. By embedding the two tonal patterns within the same frequency range, we hoped to discourage dependence on the absolute frequency of the individual tones (see D'Amato \& Salmon, 1984, Experiment 1).

\section{Procedure}

Training. All training sessions consisted of 48 trials, 24 with the positive tonal pattern (S+) and 24 with the negative pattern (S-). To initiate a trial, the subject activated a centrally placed switch 4 times, which caused the stimulus of that trial, an S+ or an Sexemplar, to be presented by the speaker under the right lever. Responses to the right lever were ineffective during the first 2.5 sec, which allowed for two full playings of the acoustic stimulus. A 3.1-sec response window followed, during which two additional playings could occur. On S+ trials, the first response registered on the right lever during the response window resulted in termination of the acoustic stimulus and delivery of a reward pellet, followed by entry into the 20 -sec ITI. Failure to respond to the right lever was an incorrect response, which resulted in a $1-$ min timeout, followed by the ITI. On S- trials, refraining from pressing the right lever during the response window was a correct response, which moved the trial to the ITI; responding during the $3.1-\mathrm{sec}$ response window constituted an incorrect response, which resulted in the 1-min timeout, followed by entry into the ITI.

Phase 1. Experience with a pilot subject indicated that training the monkeys from the outset with all of the S+ and S- exemplars was likely to result in exceedingly slow learning of the discrimination. Consequently, for Moe and Tiny, training in the high-octave frequency range began with only a single exemplar of $S-$ and $S+$, $A 1$ and D3, respectively. Boris and Tom also began training with single exemplars, but these were 3-note sequences in which the intervals spanned by successive notes were twice those of the 4-note stimuli. Thus the S- exemplar was 2688, 3378, and $4237 \mathrm{~Hz}$, and the $S+$ sequence was 4808,3788 , and $3012 \mathrm{~Hz}$. Our rationale was that the larger frequency increments and decrements might increase the salience of the tonal patterns, thereby facilitating their detection. When 42 training sessions failed to produce any sign that the two monkeys were learning the discrimination, they were shifted to the two 4-note exemplars.

After the subjects attained the criterion of at least $81 \%(39 / 48)$ correct responses in one session with the single exemplars, the other exemplars of the positive and negative tonal patterns were introduced. Training with all six discriminative stimuli continued until the same criterion was achieved. The monkeys were then shifted to the low octave range and trained with the three positive and three negative exemplars. Only Tiny was able to reach the $81 \%$ criterion within 20 sessions. Because of their poor performance levels, the other three subjects were trained with the exemplar set reduced to A1 and D3 (of the low octave) until they met the $81 \%$ correct criterion, when the full exemplar set was restored. Boris required separate training on A2 and D2 as well. All subjects ultimately achieved the $81 \%$ correct criterion with the full set of six exemplars.

Phase 2. The point of this phase was to ensure that the monkeys could simultaneously maintain the high and low octave discriminations, and that they could successfully shift from one octave range to the other. The first step was to return them to the high octave range with all exemplars; the performance criterion was increased to $83 \%$ correct $(40 / 48)$ responses. This achieved, they were then required to meet the same criterion in the low octave range, again with all exemplars. Finally, for three of the subjects the high and low octave ranges were alternated either every session or every two sessions, until they were capable of satisfying the $83 \%$ correct criterion in both octaves in two consecutive sessions. For Tiny, who was the lead animal in the study, the two octave ranges were alternated within sessions, the first 24 trials being in one octave and the second 24 trials in the other. The high octave range was presented first on alternate sessions, and the criterion was set at $85 \%$ correct in a single session, with at least $83 \%$ correct responses in both octaves.

Transfer test. The test phase consisted of 1648 -trial transfer sessions in the middle-octave frequency range. All three exemplars of the positive and of the negative tonal patterns were presented in all sessions.

\section{Results and Discussion}

\section{Training}

The results from Phases 1 and 2, individual-subject as well as group data, are presented in Table 1. In Phase 1, the monkeys required 48.0 sessions on the average to reach criterion in the high octave range with the full exemplar set (not including the $\mathbf{4 2}$ sessions received by Boris and Tom with the 3-tone stimuli). With exception made for Tiny, little savings were shown when the subjects were shifted to the low octave. Tiny's generally superior acquisition performance may be due to his extensive past experience with the very demanding acoustic discrimination mentioned above (see D'Amato \& Salmon, 1984). In Phase 2 , most of the monkeys had little difficulty achieving the slightly more stringent criterion in the high and low octaves; nevertheless, a fair amount of additional training was necessary before they could effectively shift between the octaves on a daily (or in the case of Tiny, a within-day) basis. Still, all subjects managed to do so.

\section{Transfer test}

Despite their substantial discriminative capacity in the high and low octaves, as a group the monkeys displayed little transfer when tested in the middle octave. The mean percentage of correct responses on the first test session was a mere $52.6 \%$, and at the end of 16 testing sessions it had advanced to only $66.7 \%$. Had the monkeys' discriminative performance in the high and low octaves been based importantly on the tonal pattern of the S+ and S-

\section{Table 1}

Numbers of Sessions to Criterion in Phases 1 and 2 of Training in the High, Low, and Alternating Octave Conditions

\begin{tabular}{llllcccc}
\hline & \multicolumn{2}{c}{ Phase 1 } & & \multicolumn{3}{c}{ Phase 2 } & Total \\
\cline { 2 - 4 } \cline { 5 - 6 } Subject & High & Low & & High & Low & Alt. & Sessions \\
\hline Boris* & 44 & 74 & & 1 & 2 & 44 & 165 \\
Moe & 56 & 43 & & 6 & 13 & 14 & 132 \\
Tiny & 52 & 11 & & 5 & 4 & 12 & 84 \\
Tom* & 40 & 51 & & 15 & 21 & 17 & 144 \\
Mean & 48.0 & 44.8 & & 6.8 & 10.0 & 21.8 & 131.3 \\
Median & 48.0 & 47.0 & & 5.5 & 8.5 & 15.5 & 138.0 \\
\hline
\end{tabular}

*Received 42 sessions with 3-tone stimuli. 
exemplars, a far greater degree of transfer should have been obtained, even on the initial test sessions.

Granted that immediate transfer was not obtained, did the monkeys at least show a significant degree of "savings" in their 16 test sessions in the middle octave? A reasonable way of addressing this issue is to compare the monkeys' performance on the transfer test sessions with that obtained in Phase 1 during the first 16 sessions of training in the low octave, which for all subjects took place with the full exemplar set. Figure 2 presents these data in blocks of two sessions. Because Tiny reached the low octave criterion of Phase 1 in 11 sessions, his curve for this condition is truncated and the last point is based on one session.

It is clear from Figure 2 that only Moe showed savings in the middle octave; on the other hand, quite the opposite result was produced by Tiny. Individual-subject randomized-blocks ANOVAs (based on five 2-session blocks in the case of Tiny) indicated that the difference between performance in the low and middle octaves was significant for both Moe and Tiny $[F(1,7)=29.54$, $p<.01$, and $F(1,4)=71.75, p<.01$, respectively.] In the case of Boris and Tom, the performance difference over the 16 sessions was not significant.

\section{Analysis of Exemplars}

The failure to obtain substantial transfer to the middle octave implies that the monkeys' capacity to discriminate between the $\mathbf{S}+$ and $\mathrm{S}-$ stimuli in the high and low octaves was not based to any important degree on tonal pattern. But if not tonal pattern, what acoustic cues did they use? In an effort to address this interesting issue, we analyzed the monkeys' performance separately for each of the six exemplars.

We started with the simple hypothesis that the major controlling acoustic cue was the frequency of the first tone of each exemplar. Despite the fact that each ascending and descending pair (for example, A1 and D1) was constructed from exactly the same four tones, the frequency of the first tone of an exemplar uniquely defined whether it was an $\mathrm{S}-$ or an $\mathrm{S}+$ exemplar. Thus, for the high octave range the first-tone frequencies of the $S-$ sequences $A 1, A 2$, and $A 3$ were 2688,3012 , and $3378 \mathrm{~Hz}$, respectively; they were 3788,4237 , and $4808 \mathrm{~Hz}$ for the S+ exemplars D1, D2, and D3, in that order. The positive and negative sequences could also be completely identified by the last tones of the exemplars, which for A1, A2, and $A 3$ were, in order, 3788, 4237, and $4808 \mathrm{~Hz}$; for D1, D2, and D3 they were 2688,3012 , and $3378 \mathrm{~Hz}$, respectively. Although we previously found that our monkeys are capable of distinguishing tunes on the basis of their last tone (D'Amato \& Salmon, 1984), other things being equal, first-tone differences are likely to be more salient because of the quiet period that precedes them. In any case, it is not difficult to distinguish empirically between the two possibilities.
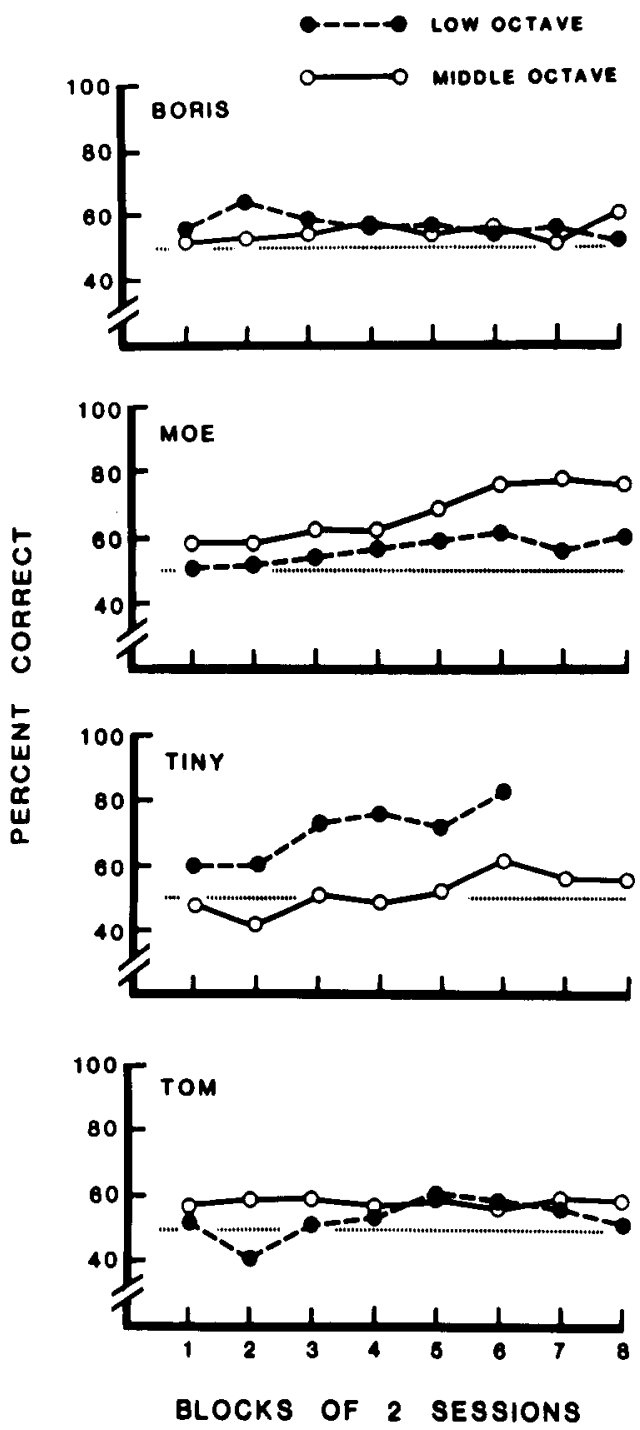

Figure 2. Percentages of correct responses for each monkey during the 16 (middle-octave range) transfer sessions of Experiment 2 in blocks of two sessions. For comparison, each subject's performance during the first 16 (11 in the case of Tiny) low-octave range sessions of Phase 1 is also shown. There were 48 trials in each session.

A strong dependence on first-tone frequency implies that performance on the $S-$ exemplars would be best with $A 1$ and worst with A3, whose initial tone is closest to the firstnote frequencies of the $S+$ exemplars. With regard to the latter sequences, performance should show an increasing trend from D1 to D3. Last-tone dependence leads to quite the opposite predictions: an increase in performance from A1 to A3, and a decreasing trend from D1 to D3.

To evaluate these different implications, we calculated for each subject the percentage of correct responses obtained with each high octave exemplar during the criterial session of Phase 1 and the two criterial sessions of Phase 2 (the second of which occurred during alternating sessions or, in the case of Tiny, during within-session alternation 
combined over two sessions). Since each of the three criterial sessions contributed eight trials to each exemplar, there were 24 trials per exemplar for each subject. A similar calculation was performed for the low octave exemplars over their three criterial sessions.

Figure 3 presents the percentages of correct responses for each exemplar in the high and low octave ranges averaged over the four subjects. The exemplars are located on the abscissa in terms of the frequency of their initial notes. As predicted by the assumption that major control was vested in the first tone of the sequences, for both the high and low octaves performance on S- exemplars generally decreased from A1 to A3 while it increased across the S+ exemplars D1, D2, and D3. To evaluate these trends statistically, a randomized-blocks ANOVA, incorporating the factors subjects, exemplar type (ascending or descending), and exemplar pair $(1,2$, or 3$)$, was performed on the group data separately for the high and low octaves. Because of the prediction that performance would decline across the ascending pairs but increase across the corresponding positive exemplars, the primary assessment of interest was the interaction between exemplar type and exemplar pair.

In the high octave analysis, exemplar type was highly significant $[F(1,15)=27.73, p<.001]$, reflecting a bias to press the response lever. Because reinforcement only followed lever presses, errors of commission (pressing the lever on $\mathbf{S}-$ trials) were more frequent than errors of omission (failing to press on $\mathrm{S}+$ trials). More important for present purposes, the interaction between exemplar type and pair was also significant $[F(2,15)=9.84$, $p<.01]$. The difference between the linear components of the ascending and descending exemplars accounted for

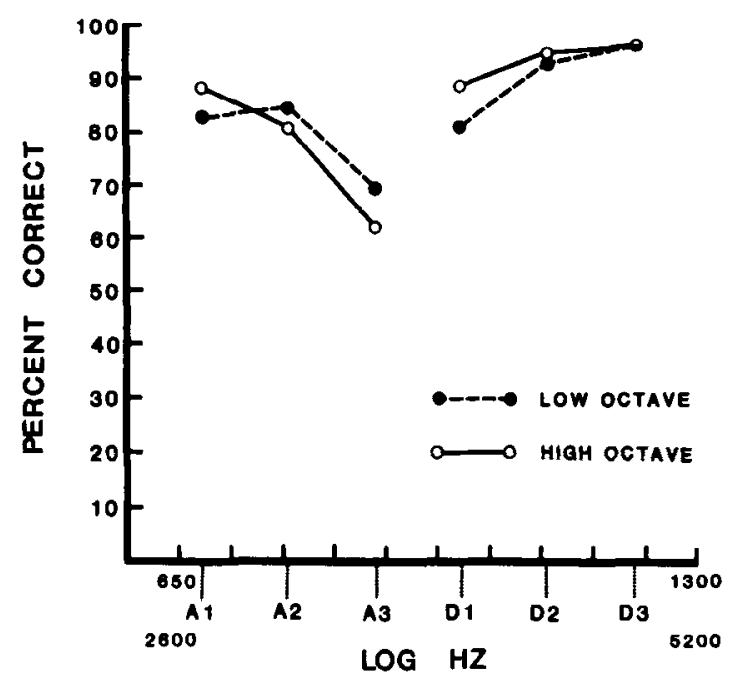

Figure 3. Percentages of correct responses for each of the six exemplars of the high-octave and the low-octave frequency ranges of Experiment 2. Each ascending exemplar $(\mathbf{A 1}, \mathbf{A 2}, \mathbf{A 3})$ and descending exemplar (D1, D2, D3) is represented in terms of the frequency of its first tone in the high octave (2600-5200 Hz scale) and low octave (650-1300 Hz scale) ranges. The data are based on three criterial sessions in the high and low octaves (see text). about $97 \%$ of the interaction variation. For the low octave data, exemplar type was again significant $[F=5.94$, $p<.05]$. The interaction between exemplar type and pair was very close to conventional significance levels $[F=3.51, p=.056]$, and once more about $97 \%$ of the interaction variation was due to the difference between the linear components of the $S$ - and $S+$ exemplars, which proved statistically significant $[F(1,15)=6.83$, $p<.05]$.

It appears plausible from these results that, for the most part, the monkeys solved the high and low octave discriminations by attending primarily to the first tone of each exemplar. There is, another aspect of the monkeys' performance that points in this direction. Recall that initially Boris and Tom were trained with a 3-tone positive and negative exemplar that spanned an interval of four whole tones; the 4-tone S+ and S- exemplars presented to Moe and Tiny spanned only three whole tones. We thought the larger frequency changes of the 3-tone exemplars would result in faster acquisition, inasmuch as they should have increased the salience of the ascending and descending patterns. They had the opposite effect. Over the last two of their 42 sessions with the 3-tone exemplars Boris and Tom averaged only $54.2 \%$ correct responses; in contrast, Moe and Tiny reached the $81 \%$ correct criterion within 32 sessions. The latter subjects may have profited from the fact that, for the two 4-tone exemplars (A1 and D3), the first two tones of one exemplar were outside the frequency range of the other.

If the high and low octave discriminations were importantly based on the first note of the exemplars, it follows that discriminative performance should be maintained at a reasonably high level if each exemplar were presented as four repetitions of the first note, rather than as ascending and descending sequences. Unfortunately, because of their assignment to a neuropsychological experiment, the present monkeys were not available for testing this important implication. However, Atom, an 8-year-old male monkey who served as the pilot subject, was available for this purpose. Following Hulse and Cynx (1985), Atom had been trained on high and low frequency ranges that were not quite two octaves apart. As a result, the lowest tone of the high frequency range was identical to the highest tone of the middle range, and the highest tone of the low frequency range was the same as the lowest tone of the middle range. Because Atom failed to show any transfer whatever to the middle frequency range, we thereafter avoided the overlapping frequency ranges.

In any case, to assess the previous implication, Atom, who was away from the task for almost 26 months, was returned to the high frequency range, and within five 48trial sessions his performance reached $85.4 \%$ correct on the full exemplar set. He was then given an additional four sessions with the ascending and descending sequences interspersed with four sessions in which only the first note of each sequence was presented, repeated four times. Although his performance was somewhat higher with the normal sequences than with the latter $-88.5 \%$ versus 
83.9\% correct, respectively - the difference was not reliable $[t(6)=1.30, p>.20]$, and the same performance level $(91.7 \%)$ was achieved on the last session of each type.

We should emphasize that we do not interpret the preceding results as implying that the first note of the ascending and descending sequences was in all cases the only discriminative cue used by the monkeys. If it were, Atom's performance should have been potentiated by the repeated notes condition (see D'Amato \& Salmon, 1984, Experiment 3). Our view is that, although the first note constituted a major discriminative stimulus, other sources of control may have, been available to the monkeys. For example, they may have discriminated the pitch sequence of the first two notes. As pointed out elsewhere (D'Amato, 1988), such discriminations do not necessarily imply tonal pattern perception.

\section{GENERAL DISCUSSION}

The degree of insensitivity of our monkeys to tonal pattern is surprising in view of the well-developed auditory cortex of cebus monkeys and the complexity of their auditory communication system (see, e.g., Robinson, 1984). Although one must be appropriately cautious about the generality of negative instances, we think it fair to conclude from our present and previous research that tonal pattern perception of non-species-specific acoustic stimuli is at best poorly developed in cebus, and perhaps in other monkeys as well. Extensive past experience in discriminating, matching, and retaining acoustic stimuli did little to promote tonal pattern perception. Multiple exemplars of the simplest tonal patterns, unidirectional frequency contours, were also without effect, even with very extensive training. We calculate that in Experiment 2 the minimum number of exposures to the positive and negative tonal patterns ranged from about 8,000 each for Tiny to some 16,000 each for Boris. D'Amato and Salmon (1984) estimated that the monkeys of their Experiment 3 were exposed to the two tunes some 25,000 times each, without displaying evidence of tonal pattern perception. It is possible, of course, that more refined techniques may produce evidence for frequency contour detection in monkeys, but we doubt that this capacity will be shown to assume the dominant position in the processing of acoustic stimuli that it enjoys in humans.

As suggested previously (D'Amato, 1988), the possibility exists that in monkeys, and perhaps in other animals as well, the processing of frequency contour is largely confined to species-specific signals. Japanese macaque monkeys were reported to learn a discrimination based on a communicatively relevant feature of species-specific acoustic stimuli faster than a discrimination based on a communicatively irrelevant feature. Moreover, the monkeys displayed a right-ear advantage while engaged in the former task, but not while engaged in the latter (Beecher, Peterson, Zoloth, Moody, \& Stebbins, 1979). These results suggest that species-specific acoustic signals may be capable of engaging auditory processing mechanisms, perhaps including those responsible for frequency contour extraction, denied to other acoustic stimuli. It remains for future research to determine whether there is any substance to this suggestion.

Species other than monkeys have been found wanting with regard to their capacity to extract tonal pattern from non-species-specific acoustic stimuli. The rat subjects in the D'Amato and Salmon (1984) study performed much as the monkeys with regard to basing their discrimination between the two tunes on local cues. Hulse and his colleagues have extensively studied tonal pattern perception in songbirds, with meager results. Like the monkeys, the birds failed to transfer an ascending/descending pattern discrimination to exemplars displaced by an octave (Hulse \& Cynx, 1985, Experiment 1), or even to transpositions much less than an octave (Cynx, Hulse, \& Polyzois, 1986), and they showed no transfer to the middle octave in an experiment that served as the model for our Experiment 2 (Hulse \& Cynx, 1985, Experiment 2). The birds did transfer to "novel" exemplars within the training octave, but it is difficult with such "near" transfer tests to rule out stimulus generalization and nonrelational perceptual processes as contributing factors (D'Amato, 1988). Parakeets, too, seem insensitive to tonal patterns of non-species-specific stimuli (Dooling, Brown, Park, Okanoya, \& Soli, 1987).

Interestingly, Cynx et al. (1986) found that starlings trained to discriminate between ascending and descending sequences maintained the discrimination when tested with each exemplar's first tone repeated four times. However, unlike the results obtained from Atom, the difference between performance with the test tones and performance with the patterned sequences was significant. The authors recognized that the higher performance with the patterned sequences did not necessarily imply tonal pattern perception. They pointed out that the birds could have extracted absolute frequency information from notes other than the first. Another possibility is that the pitch perception of an exemplar's first note is somewhat different when it occurs in conjunction with the other notes of the sequence than when it occurs alone or is repeated. When, for example, our high-octave A1 ascending sequence was played repeatedly and the observer's attention was focused on the first note, the relatively high pitch of the last note of the sequence heard from the previous playing sometimes resulted in a pitch perception of the subsequent first note that was lower than the pitch produced by the same first note repeated four times. Perceptual interactions of this sort need to be taken into account when evaluating the significance of less than robust evidence for tonal pattern perception in animals.

In summary, it is surprising that tonal pattern perception of non-species-specific acoustic stimuli is so poorly represented in animals. There is reason to believe that dolphins may possess this capacity to some degree (see Richards, Wolz, \& Herman, 1984), as well as pygmy chimpanzees (Savage-Rumbaugh, McDonald, Sevcik, 
Hopkins, \& Rubert, 1986), but much more information is needed regarding its representation in these and in other species. As many have suggested, there may be an intimate connection between the capacity for tonal pattern perception and that for acoustically based language. Perhaps they tap common processing structures, accounting for the representation of both in human infants (Papousek \& Papousek, 1981) and the rudimentary nature of tonal pattern perception in many nonhuman species.

\section{REFERENCES}

Beecher, M. D., Peterson, M. R., Zoloth, S. R., Moody, D. B., * Stebbins, W. C. (1979). Perception of conspecific vocalizations by Japanese macaques. Brain, Behavior \& Evolution, 16, 443-460.

Cheney, D., Seyfarth, R., Smuts, B. (1986). Social relationships and social cognition in nonhuman primates. Science, 234, 1361-1366.

Colombo, M., D D'Aмato, M. R. (1986). A comparison of visual and auditory short-term memory in monkeys (Cebus apella). Quarterly Joumal of Experimental Psychology, 38B, 425-448.

CynX, J., Hulse, S. H., Polyzors, S. (1986). A psychophysical measure of pitch discrimination loss resulting from a frequency range constraint in European starlings (Sturnus vulgaris). Joumal of Experimental Psychology: Animal Behavior Processes, 12, 394-402.

D'AMATo, M. R. (1988). A search for tonal pattern perception in cebus monkeys: Why monkeys can't hum a tune. Music Perception, $5,453-480$.

D'Amato, M. R., Colombo, M. (1985). Auditory matching-to-sample in monkeys (Cebus apella). Animal Leaming \& Behavior, 13, 375-382.

D'Amato, M. R., SAlmon, D. P. (1982). Tune discrimination in monkeys (Cebus apella) and in rats. Animal Learning \& Behavior, 10, 126-134.

D’Amato, M. R., Salmon, D. P. (1984). Processing of complex auditory stimuli (tunes) by rats and monkeys (Cebus apella). Animal Learning \& Behavior, 12, 184-194.

Dooling, R. J., Brown, S. D., Park, T. J., Okanoya, K., \& Soli,
S. D. (1987). Perceptual organization of acoustic stimuli by budgerigars (Melopsittacus undulatus). Joumal of Comparative Psychology, 101, 139-149.

HulsE, S. H., \& CYNX, J. (1985). Relative pitch perception is constrained by absolute pitch in songbirds (Mimus, Molothrus, \& Stumus). Journal of Comparative Psychology, 99, 176-196.

Papousex, M., \& Papousek, H. (1981). Musical elements in the infant's vocalizations: Their significance for communication, cognition, and creativity. In L. P. Lipsitt (Ed.), Advances in infant research, (Vol. 1, pp. 163-224). Norwook, NJ: Ablex

Richards, D. G., Wolz, J. P., \&e Herman, L. M. (1984). Vocal mimicry of computer-generated sounds and vocal labeling of objects by a bottlenosed dolphin, Tursiops truncatus. Journal of Comparative Psychology, 98, 10-28.

Robinson, J. G. (1984). Syntactic structures in the vocalizations of wedge-capped capuchin monkeys, Cebus olivaceus. Behaviour, 90, 46-79.

Savage-Rumbaugh, S., McDonald, K., Sevcik, R. A., Hopkins, W. D., RuBert, E. (1986). Spontaneous symbol acquisition and communicative use by pygmy chimpanzees (Pan paniscus). Journal of Experimental Psychology: General, 115, 211-235.

Seyfarth, R. M., Cheney, D. L., \& Marler, P. (1980). Monkey responses to three different alarm calls: Evidence of predator classification and semantic communication. Science, 210, 801-803.

\section{NOTE}

1. Young cebus monkeys are notoriously difficult to sex correctly solely by visual inspection of the caged animal. Moe was represented by the supplier to be a male and was reported as such in previous publications. But the sexual dimorphism in size and weight that accompanies maturation in this species seemed to place him on the wrong side of the ledger. Recently a close physical inspection conducted during a surgical procedure confirmed what probably was already known to the other monkeys in the colony: Moe is a female.

(Manuscript received April 4, 1988; revision accepted for publication June 29,1988 .) 